Recibido: 16/06/2020 --- Aceptado: 17/12/2020 --- Publicado: 12/04/2021

\title{
DIMENSIONES MOTIVACIONALES EN FACEBOOK EN ESTUDIANTES Y TRABAJADORES UNIVERSITARIOS DEL ECUADOR
}

\section{MOTIVATIONAL DIMENSIONS ON FACEBOOK IN UNIVERSITY STUDENTS AND WORKERS FROM ECUADOR}

\section{Álvaro Jiménez-Sánchez ${ }^{1}$. Universidad Técnica de Ambato. Ecuador.} al.jimenez@uta.edu.ec

Eliza Carolina Vayas Ruiz. Universidad Técnica de Ambato. Ecuador. elizacvayasr@uta.edu.ec

\section{Cómo citar el artículo:}

Jiménez-Sánchez, A. y Vayas Ruiz, E. C. (2021). Dimensiones motivacionales en Facebook en estudiantes y trabajadores universitarios del Ecuador. Revista de Comunicación de la SEECI, 54, 43-63. http://doi.org/10.15198/seeci.2021.54.e656

\section{RESUMEN}

Conocer los usos y gratificaciones que los individuos hacen de Facebook es importante para saber por qué la utilizan y cómo debería proceder el marketing empresarial. Esta investigación se centra en analizar las posibles dimensiones motivacionales y su relación con la satisfacción y uso de la plataforma. Se suministró un cuestionario a 214 estudiantes de 7 carreras distintas y 50 trabajadores de la Universidad Técnica de Ambato (Ecuador). El análisis factorial y correlacional mostraron diversos componentes en función de la edad, así como relaciones con el grado de satisfacción o el tiempo de conexión. Los resultados indicaron diferencias estadísticamente significativas entre los usos que hacen los estudiantes en comparación con los trabajadores, así como también discrepancias en función del género y la edad. Las conclusiones conducen a un estudio más profundo y longitudinal de la plataforma, proponiendo nuevas vías de investigación, así como diferentes reflexiones sobre los usos y repercusiones que este sitio de red social tiene cada día en la vida de millones de personas.

1 Álvaro Jiménez Sánchez: Doctor en Comunicación. Universidad de Salamanca. Docenteinvestigador de la Facultad de Jurisprudencia y Ciencias Sociales de la Universidad Técnica de Ambato. al.jimenez@uta.edu.ec 
PALABRAS CLAVE: Facebook - Motivaciones - Estudiantes - Trabajadores - Redes sociales.

\section{ABSTRACT}

To know the uses and gratifications that individuals make of Facebook is important to learn why they use it and how business marketing should proceed. This research focuses on analyzing the possible motivational dimensions and their relationship with satisfaction and use of the platform. A questionnaire was provided to 214 students from 7 different careers and 50 workers from the Technical University of Ambato (Ecuador). The factorial and correlational analysis showed various components depending on age, as well as relationships with the degree of satisfaction or connection time. The results indicated statistically significant differences between the uses made by students compared to workers, as well as discrepancies based on gender and age. The conclusions lead to a more profundity and longitudinal study of the platform, proposing new lines of research, as well as different reflections on the uses and repercussions that this social network site has every day in the lives of millions of people.

KEYWORDS: Facebook - Motivations - Students - Workers - Social Networks.

\section{DIMENSÕES MOTIVACIONAIS NO FACEBOOK EM ESTUDANTES E TRABALHADORES UNIVERSITÁRIOS NO EQUADOR}

\section{RESUMO}

Conhecer os usos e gratificações que os indivíduos fazem do Facebook é importante para saber porque o utilizam de como deveria proceder o marketing empresarial. Esta pesquisa se centra em analisar as possíveis dimensões motivacionais e sua relação com a satisfação e o uso da plataforma. Se fornece um questionário a 214 estudantes de 7 graduações diferentes e 50 trabalhadores da Universidade Técnica de Ambato (Equador). A análise fatorial e correlacional mostrou diversos fatores em função da idade, assim como as relações com o grau de satisfação ou o tempo de conexão. Os resultados indicam diferenças estatisticamente significativas entre a forma de uso dos estudantes em comparação com os trabalhadores, assim como discrepâncias em função do gênero e da idade. As conclusões levam a um estudo mais aprofundado e longitudinal da plataforma, propondo novas formas de pesquisa, assim como diferentes reflexões sobre os usos e repercussões que este site de rede social tem cada dia na vida de milhões de pessoas.

PALAVRAS CHAVE: Facebook - Motivações - Estudantes - Trabalhadores- Redes sociais.

\section{INTRODUCCIÓN}

Facebook se origina en el 2004 como un lugar virtual para estudiantes de la Universidad de Harvard. La idea fue tan exitosa que el proyecto hasta la fecha es el sitio web de redes sociales más empleado en todo el mundo con más de dos mil millones de usuarios (We are social y Hootsuite, 30-01-2019). El creciente volumen 
de negocio ha hecho que esta empresa pase a la historia en el 2012 por ser la tecnológica con mayor salida a bolsa (20minutos.es, 18/05/2012). Su expansión mercantil le ha permitido adquirir aplicaciones informáticas tan importantes como Messenger, Instagram o WhatsApp (El Mundo, 01/02/2017).

En el 2017 incorporó a setenta millones de usuarios activos en un solo trimestre, algo que nunca antes había alcanzado y que obliga aún más a los responsables de las redes sociales de las empresas a incluirla en las estrategias fundamentales del Social Media Marketing (Mejía, 02/05/2017). Con más de treinta y siete mil empleados a finales de marzo de 2019 (Facebook-Newsroom, 2019), se financia principalmente de unas ventas por publicidad en continuo crecimiento, especialmente en dispositivos móviles (El Mundo, 01/02/2017).

El negocio de momento sigue en expansión, cada vez más personas integran esta plataforma a su cotidianidad, de ahí que muchos investigadores de diversas áreas hayan puesto atención al estudio de las redes sociales para intentar comprender las prácticas de los usuarios, las implicaciones que estas tienen en la cultura y el significado de este tipo de aplicaciones (Boyd y Ellison, 2007; Salzinger, Antrobus y Hammer, 2015). Por tanto, uno de los objetivos principales tanto de Facebook como de las empresas e instituciones que lo han incorporado a su funcionamiento, sería justamente analizar cómo y por qué usan esta plataforma, y observar su evolución a lo largo de los años según patrones como la edad, el género o la ocupación.

Los mayores consumidores de las redes sociales son los jóvenes (18 a 34 años), donde un $90 \%$ las utiliza habitualmente (Colas-Bravo, González-Ramírez y de PablosPons, 2013; We are social y Hootsuite, 30/01/2019). Este dato coincide con la población ecuatoriana; si se compara el número de horas dedicadas a este tipo de redes en función del segmento de edad, los jóvenes son los que más tiempo le dedican en el país andino (García-Guerrero, 2015).

Latinoamérica es una de las regiones del mundo con más actividad en las redes sociales, siendo Facebook la de mayor incidencia y con mucha diferencia respecto al resto de plataformas digitales (Hutchinson, 2016). En Ecuador, la incursión de las nuevas tecnologías ha crecido considerablemente en los últimos años, aunque todavía presenta una ligera brecha digital entre los jóvenes y los adultos (JiménezSánchez et al., 2019). A 2020, el país andino cuenta con unos doce millones de habitantes que usan activamente las redes sociales digitales (69\% de la población), donde Facebook se sitúa como la séptima web más visitada y de las primeras en tiempo de uso. El dispositivo más empleado para acceder es el móvil (96,8\%). El resto de plataformas como Instagram, Linkedin, TikTok, Twitter, Pinterest o Snapchat están todavía lejos de sus cifras, aunque en los últimos años Instagram ha crecido con bastante rapidez hasta situarse en los cuatro millones de usuarios (DataReportal, 2020; Dávalos, 14/03/2020).

Debido a estas cifras y a la repercusión en la vida de sus usuarios, se hace conveniente ahondar en las diferentes motivaciones que subyacen a esta plataforma virtual. 


\subsection{Antecedentes}

Mark Zuckerberg no fue el primero en trabajar las redes sociales virtuales y desde sus inicios tuvo que competir con otras de gran importancia como Friendster, MySpace, MSN Spaces, Hi5, Tuenti, Fotolog, Buzz, o SecondLife por mencionar algunas con el similar propósito pero con diferentes resultados. Maslow (1970) teorizaba sobre la necesidad de aceptación social como parte fundamental del ser humano y su importancia en la autoestima. Con Internet surgieron plataformas que trataron de favorecer esta sociabilidad, pero ha sido Facebook quien hasta la fecha ha llegado a la hegemonía mundial.

Según su fundador, una de las claves del éxito empresarial es crear valor, originar productos que sean innovadores y valiosos para sus usuarios (Gutiérrez, 19/09/2013), esto significa centrar esfuerzos por conocer las necesidades de los clientes, sus particularidades, motivaciones, y así poder ir evolucionando a medida que estas van cambiando con el contexto.

Numerosas investigaciones se han centrado específicamente en estudiar el comportamiento de las personas en las redes sociales virtuales desde la teoría de los usos y gratificaciones (Katz, Blumler y Gurevitz, 1986), concluyendo que las utilizan para satisfacer una serie de necesidades como la aceptación social (Flanagin y Metzger, 2001; Lee, 2009; Papacharissi y Rubin, 2000) y lograr diferentes objetivos motivacionales como poder relacionarse de otra manera con los amigos y compañeros (García, López de Ayala y Gaona, 2012). Así mismo, los usos que se hacen dependen de factores como la edad, el sexo o el tiempo de uso (García et al., 2012; Igartua y Rodríguez de Dios, 2016; Joison, 2008; Sheldon, 2008; Spiliotopoulos y Oakley, 2013).

Si bien la mayoría recurre a las redes sociales para comunicarse y estar en contacto con la familia y amigos (Colás-Bravo, González-Ramírez y Pablos-Pons, 2013; Raacke y Bonds-Raacke, 2008; Sheldon, 2008; Urista, Dong y Day, 2009), el predictor más importante en el uso del Facebook no es tanto la conexión social sino la actualización del estado. Esto quiere decir que no importa tanto el número de contactos como el hecho de tener una actitud activa lo que determina la utilización de esta plataforma (Alhabash, Park, Kononova, Chiang y Wise, 2012).

Dentro de los motivos más comunes a la hora de usar esta red social destacan pasar el tiempo y la dimensión de entretenimiento para relajarse (Igartua y Rodríguez de Dios, 2016; Papacharissi y Mendelson, 2011; Smock et al., 2011). A su vez, Facebook estaría determinado principalmente por las necesidades básicas de pertenencia y auto-presentación (Nadkarni y Hofmann, 2012), conceptos de gran importancia tal y como describiera Maslow (1970) en su jerarquía de necesidades propuesta. Otras dimensiones motivacionales de interés aportadas por estas investigaciones serían la pertenencia a una comunidad virtual, el carácter de coolness, el mantenimiento de las relaciones o la compañía, todas ellas relacionadas 
directa o indirectamente con este sentido de pertenencia que Facebook trataría de cubrir como propósito primordial junto al entretenimiento generado.

En los últimos años, los jóvenes usuarios de este tipo de redes sociales siguen motivados en utilizarlas para cubrir sus necesidades sociales y personales, tales como estar comunicados, informados, conocer qué hacen sus contactos o promocionar una versión narcisista y feliz de sus propias vidas (Tarullo, 2020). Sin embargo, plataformas como TikTok o Instagram han irrumpido con fuerza en estos años al mostrarse igual de capaces o incluso más efectivas a la hora de cubrir estas necesidades personales y sociales (Kim y Kim, 2019; Morais-Martín, 2020), especialmente en los jóvenes usuarios (Ahlse, Nilsson y Sandström, 2020; CallejoGonzález, 2019; Sheldon y Newman, 2019)

Por último, se encuentran diferencias según el contexto cultural (Alhabash et al., 2012; Kim, Sohn y Choi, 2011) donde la utilización de Facebook vendría determinada por la situación espacio-temporal y evolutiva de las personas, lo que implica tratar de validar transculturalmente el modelo de usos y gratificaciones asociadas al Facebook (Nadkarni y Hofmann, 2012), pues cada cultura ha demostrado un empleo diferente de la plataforma, lo que deriva a investigar este fenómeno social en otros lugares o contextos culturales (Igartua y Rodríguez de Dios, 2016), como, por ejemplo, el poco investigado panorama iberoamericano (Salzman, 2015).

\section{OBJETIVOS}

Así, el objetivo principal de esta investigación es analizar la satisfacción con la plataforma y su relación con las distintas dimensiones motivacionales, diferenciando por un lado entre estudiantes y trabajadores, y por otro, posibles discrepancias entre el género y la edad.

Los resultados derivados pueden ser relevantes para cualquier empresa interesada en utilizar Facebook para su actividad comercial tal y como propone la propia plataforma (Facebook para empresas, 2019). El propósito final es seguir aportando en la construcción de un mapa geo-cultural que permita comprender la satisfacción y las motivaciones por la plataforma.

\section{METODOLOGÍA}

Durante el periodo académico 2016-2017 se suministra un cuestionario a 214 estudiantes y 50 trabajadores de la Universidad Técnica de Ambato (centro de Ecuador) con perfil en Facebook con la intención de medir las variables relacionadas con su uso. El $54,9 \%$ hombres y el $43,6 \%$ mujeres (1,5\% no indicaron). El rango de edad de los estudiantes es de 17 a 28 años (Media $=20$ ) y el de los trabajadores de 25 a 58 años (Media $=36$ ). Dado que la población total estudiantil y trabajadora universitaria es de 18.000 (16.000 y 2.000 respectivamente), se obtiene una muestra mínima de 263 personas con un nivel de confianza del $95 \%$ y un margen de error del $6 \%$. El tipo de muestreo a utilizar es por conglomerados bietápico, donde primero se definen las distintas carreras a participar (Ingeniería de sistemas, Ingeniería 
mecánica, Cultura física, Bioquímica, Diseño gráfico, Contabilidad y Auditoría, Comunicación social) y posteriormente se aplica el muestreo aleatorio simple para la selección de los cursos.

Así, se realizó una encuesta a una clase o paralelo por carrera (siete en total) y a trabajadores de diferentes áreas (especialmente profesores y personal de administración universitaria). Todos fueron informados del propósito de la investigación y del carácter anónimo del cuestionario que debían responder de manera voluntaria. El tiempo de realización de la encuesta fue de 7 minutos aproximadamente.

Dicho cuestionario se basa en el estudio previo de Igartua y Rodríguez de Dios (2016), el cual también toma como referencias otras investigaciones, y en especial la de Sheldon (2008). El grado de satisfacción se valora del 1 (mínimo) al 10 (máximo), mientras que para los usos se emplea una escala tipo Likert del 1 (mínima motivación) al 5 (máxima).

Los ítems se adaptan al contexto lingüístico ecuatoriano, con la intención de extraer datos sobre las diversas motivaciones en el uso del Facebook, la manera de utilizar esta red social y su satisfacción para con la plataforma. Para validar dicha adaptación se recurre primeramente a un pre-test o cuestionario de prueba con 30 alumnos, tras realizarlo individualmente se procede a una fase de discusión sobre la comprensión de los ítems y sugerencias. Por último, se analiza el alfa de Cronbach de esta primera muestra para descartar aquellas cuestiones que inciden en una menor fiabilidad. Así, el instrumento de evaluación definitivo tiene un alfa de ,930 para los estudiantes y de, 944 para los trabajadores.

Posteriormente, se analizan los datos con el programa estadístico SPSS (Versión 23 para Windows). Para comprobar las posibles diferencias entre estudiantes y trabajadores se aplican las correlaciones de Pearson para las variables cuantitativas y la U-Mann-Whitney para las ordinales. Después se aplica un análisis factorial de componentes principales para reducir la dimensionalidad del conjunto de datos y por último la t-Student para ver la diferencia de los factores extraídos en estudiantes y personas empleadas.

La investigación cumple con las normas marcadas por el Comité de Ética de la Investigación y Docencia de la Universidad Técnica de Ambato.

\section{ANÁLISIS Y DISCUSIÓN}

\subsection{Resultados}

De manera general, los datos muestran que la satisfacción con Facebook es relativamente alta (7,58 en estudiantes y 6,28 en los empleados sobre diez). El mayor predictor de la satisfacción con la plataforma en estudiantes es el número de contactos. También se observa que algunos motivos de uso predicen una mayor 
satisfacción, un mayor número de contactos y un aumento en el tiempo de conexión diaria.

Debido a las diferencias encontradas en el uso del Facebook entre estudiantes y trabajadores, es conveniente mostrar los análisis por separado y posteriormente observar las divergencias entre ambos grupos.

\subsubsection{Estudiantes}

Los resultados muestran una media de 931 contactos, 233 minutos de conexión al día, 41 minutos de conexión puntual, 7,58 de grado de satisfacción y un 5,84 de grado sobre diez en el que lo echarían de menos si de pronto desapareciese esta plataforma social.

A su vez, el 57\% usa el dispositivo telefónico celular para conectarse como opción principal (de los cuales el 39\% tienen datos contratados), mientras que el resto usaría computadoras u otros dispositivos informáticos. El lugar de conexión habitual es la casa (63\%), seguido por la universidad con un $36 \%$, mientras que solo un $1 \%$ lo hace en sitios como el parque o el ciber.

En la Tabla 1 se pueden observar las correlaciones de Pearson en el uso del Facebook. El sentido de realizar estos análisis es comprobar de manera significativa la relación entre la satisfacción con la plataforma respecto al número de contactos y tiempo de uso de la misma. Siguiendo la línea de Igartua y Rodríguez de Dios (2016), se espera encontrar una alta correlación entre dichas categorías.

Tabla 1: correlaciones de Pearson entre contactos, satisfacción y tiempo de conexión.

\begin{tabular}{|l|l|l|l|l|}
\hline & $\begin{array}{l}\text { Minutos al día de } \\
\text { conexión }\end{array}$ & $\begin{array}{l}\text { Minutos de una } \\
\text { conexión }\end{array}$ & Satisfacción & Echar de menos \\
\hline Contactos &, 135 &, 107 &, $227^{* *}$ &, $226^{* *}$ \\
\hline $\begin{array}{l}\text { Minutos al día de } \\
\text { conexión }\end{array}$ &, $283^{* *}$ &, 118 &, $162^{*}$ \\
\hline $\begin{array}{l}\text { Minutos de una } \\
\text { conexión }\end{array}$ & &, 057 &,- 068 \\
\hline Satisfacción & & &, $316^{* *}$ \\
\hline
\end{tabular}

Fuente: elaboración propia.

Nota: $* p .<, 05 ; * * p .<, 01$.

Tras realizar la prueba $U$ Mann-Whitney, no se encontraron diferencias significativas en función del género respecto al uso del Facebook (contactos, minutos y satisfacción) pero sí con las edades, donde las correlaciones de Pearson más significativas muestran cómo, a medida que aumenta la edad, el número de contactos es menor $(r=-, 229$, p. $<, 01)$ y se reduce la satisfacción $(r=-, 149$, p. $<$ 
,05). Esto corrobora la correlación encontrada entre el número de contactos y el grado de satisfacción $(r=, 227, p .<, 01)$, ya que a mayor edad, menos contactos y menos satisfacción por la red social, y viceversa.

A continuación se realizó un análisis factorial de componentes principales con rotación Varimax (KMO = ,886; Bartlett $\mathrm{p} .<, 000)$. Los resultados convergen en siete factores con autovalores mayores a uno, los cuales explicaban un $63,24 \%$ de la varianza total. En las Tablas 2 y 3 se muestran los factores con saturaciones superiores a ,525, la varianza que explica cada uno, los valores de consistencia interna (Alpha de Cronbach) y datos descriptivos (media y desviación típica).

Dado el tamaño muestral y las características de las puntuaciones obtenidas, se consideró aceptable el uso de estadísticos paramétricos (Pardo y San Martín, 1998). Dependiendo de si se cumplía o no el supuesto de homocedasticidad, se tomaron en consideración los estadísticos, grados de libertad y niveles de significación asumiendo que las varianzas eran o no iguales, según el caso. Cuando se determinó la existencia de diferencias estadísticamente significativas se calculó el tamaño del efecto, de acuerdo con los procedimientos y consideraciones apropiadas al caso (Cohen, 1988; Hedges y Olkin, 1984).

Tabla 2: componentes principales en estudiantes (1).

\begin{tabular}{|c|c|c|c|}
\hline Factores (ítems de la escala) & Peso factorial & Varianza explicada & $a$ \\
\hline 1-Coolness y Compañía $(M=2,06, D T=1,16)$ & & 31,92 & , 88 \\
\hline Para conquistar & ,763 & & \\
\hline Para no sentirme solo & ,743 & & \\
\hline Porque me hace parecer coo/entre mis amigos & 706 & & \\
\hline Porque es cool & ,699 & & \\
\hline Para mantener relaciones sentimentales & ,667 & & \\
\hline Para hacer nuevos amigos & ,599 & & \\
\hline Para encontrar compañía & ,595 & & \\
\hline 2-Auto-expresión $(M=2,20, D T=1,23)$ & & 9,23 & , 87 \\
\hline $\begin{array}{l}\text { Para expresar mis opiniones sobre política o temas } \\
\text { sociales }\end{array}$ & ,728 & & \\
\hline $\begin{array}{c}\text { Para publicar informaciones sobre mis intereses } \\
\text { personales }\end{array}$ & ,695 & & \\
\hline Para contar en mi muro cosas que me pasan/han pasado & ,604 & & \\
\hline Para sentirme visibilizado & ,576 & & \\
\hline Para expresar cómo me siento &, 571 & & \\
\hline Para promocionar & ,532 & & \\
\hline
\end{tabular}

Fuente: elaboración propia. 
Se puede observar en las Tablas 2 y 3 que la dimensión motivacional más importante para usar Facebook es el mantenimiento de relaciones $(M=3,94)$, seguido del entretenimiento $(M=3,08)$, sin embargo son los componentes 1 y 2 (Coolness-Compañía y Auto-expresión I) los que mayor varianza explican (31 y 9 respectivamente), a pesar de tener menor media que los anteriores.

Tabla 3: componentes principales en estudiantes (2).

\begin{tabular}{|c|c|c|c|}
\hline Factores (ítems de la escala) & Peso factorial & Varianza explicada & a \\
\hline 3-Mantenimiento de relaciones $(M=3,94, D T=1,05)$ & & 7,05 & , 83 \\
\hline Para estar en contacto con mis amigos & ,873 & & \\
\hline Para comunicarme con mis amigos & ,846 & & \\
\hline Para mandar un mensaje a un amigo & ,838 & & \\
\hline Para chatear & ,616 & & \\
\hline 4-Entretenimiento $(M=3,08, D T=1,09)$ & & 4,30 & ,77 \\
\hline Porque es entretenido & ,746 & & \\
\hline Para ocupar mi tiempo libre & ,710 & & \\
\hline Para pasar un buen rato & ,697 & & \\
\hline Porque disfruto mucho conectándome & ,696 & & \\
\hline $\begin{array}{l}\text { 5-Auto-expresión II y componente lúdico } \\
\qquad(M=2,61, D T=1,23)\end{array}$ & & 4,06 & ,66 \\
\hline Para subir fotos o vídeos & ,655 & & \\
\hline Para poner comentarios (post) en el muro de mis amigos & ,584 & & \\
\hline Para jugar & ,530 & & \\
\hline 6-Educación $(M=3,03, D T=1,24)$ & & 3,38 & ,61 \\
\hline Para informarme & ,755 & & \\
\hline Para estudiar & ,669 & & \\
\hline 7-Comunidad virtual $(M=2,80, D T=1,16)$ & & 3,28 & ,70 \\
\hline Para ver fotos/vídeos de otras personas & ,737 & & \\
\hline Para ver el perfil de otras personas & ,713 & & \\
\hline
\end{tabular}

Fuente: elaboración propia.

Respecto al género, solo se encuentran diferencias estadísticamente significativas en el factor 1 (Coolness-Compañía), con un tamaño del efecto bajo según la prueba $t$ de Student $(\mathrm{d}=, 30 ; \mathrm{t}(193)=2,13, \mathrm{p} .=, 034)$, indicando que en los hombres predomina más este tipo de uso respecto a las mujeres. Por otra parte, se haya relación entre la edad y el quinto factor (Auto-expresión II y componente lúdico) con una correlación de Pearson de -,143 ( $\mathrm{p} .<, 05)$, dando a entender que los más jóvenes son quienes están más motivados por este tipo de uso.

Se encontraron correlaciones significativas entre el factor 3 y 4 y el grado de satisfacción $(r=, 173$, p. $<, 05$ [Mantener relaciones]; $r=, 427$, p. $<, 01$ 
[Entretenimiento]). También entre el número de contactos y los factores $4(r=, 172$, p. < ,05) y 5 [Auto-expresión II y componente lúdico] $(r=, 190, p .<, 01)$, y además, entre el cuarto componente y el número de minutos de conexión diaria ( $r=$ 179, p. < ,05), confirmando de esta manera los resultados obtenidos por Igartua y Rodríguez (2016), en el que el entretenimiento sería uno de los mayores predictores a la hora de usar Facebook.

A su vez, la fiabilidad del test $(a=, 93)$ y los datos de cada uno de los factores mostrados, validan la adaptación de los cuestionarios recurridos (Igartua y Rodríguez de Dios, 2016; Sheldon, 2008).

Por último, cabe mencionar que no se encontraron correlaciones estadísticamente significativas entre tener pareja y el resto de factores y variables propuestas, pero sí entre tener Internet en casa y el número de contactos $(r=, 253, p .<, 01)$, aunque ni el tiempo de conexión diaria y puntual ni el grado de satisfacción estuvieron relacionados lo suficiente con el hecho de disponer de Internet en el hogar. Aun así, esta información requeriría de una muestra más amplia, pues menos de 14 personas argumentaban no tener conexión en su casa.

\subsubsection{Trabajadores}

De los 50 empleados se obtuvieron 9 factores con autovalores mayores a uno, explicando un $78,15 \%$ de la varianza $(\mathrm{KMO}=, 645$; Bartlett p. $<, 000)$ y siendo el tercer componente el uso del Facebook para trabajar (7,41\% de varianza).

Tabla 4: componentes principales en trabajadores (1).

\begin{tabular}{|c|c|c|c|}
\hline Factores (ítems de la escala) & $\begin{array}{c}\text { Peso } \\
\text { factorial }\end{array}$ & $\begin{array}{l}\text { Varianza } \\
\text { explicada }\end{array}$ & a \\
\hline Factor $1(M=2,38, D T=1,14)$ & & 37,51 & ,91 \\
\hline Para ocupar mi tiempo libre & 799 & & \\
\hline Para subir fotos o vídeos & 678 & & \\
\hline Porque es entretenido & 656 & & \\
\hline Porque disfruto mucho conectándome & ,605 & & \\
\hline Para poner comentarios (post) en el muro de mis amigos & ,588 & & \\
\hline Para pasar el tiempo cuando estoy aburrido & ,562 & & \\
\hline Para pasar un buen rato & ,561 & & \\
\hline Factor $2(M=1,29, D T=, 67)$ & & 8,96 & 89 \\
\hline Para conquistar & ,847 & & \\
\hline Para no sentirme solo & ,799 & & \\
\hline Para mantener relaciones sentimentales & ,771 & & \\
\hline Para encontrar compañía & ,766 & & \\
\hline Factor $3(M=1,98, D T=1,32)$ & & 7,41 & 87 \\
\hline Para estudiar & 870 & & \\
\hline
\end{tabular}


Jiménez-Sánchez, A. y Vayas Ruiz, E C. Dimensiones motivacionales en Facebook en estudiantes y trabajadores universitarios del Ecuador

\begin{tabular}{|c|c|c|c|}
\hline Para expresar mis opiniones sobre política o temas sociales & 776 & \multirow[b]{4}{*}{5,72} & \multirow[b]{4}{*}{,919 } \\
\hline Para publicar informaciones sobre mis intereses personales & 706 & & \\
\hline Para trabajar & \multirow[t]{2}{*}{ 657 } & & \\
\hline Factor $4(M=3,1, D T=1,21)$ & & & \\
\hline Para estar en contacto con mis amigos & 880 & & \\
\hline Para comunicarme con mis amigos & 847 & & \\
\hline Para mandar un mensaje a un amigo & ,711 & & \\
\hline Para chatear & 619 & & \\
\hline
\end{tabular}

Fuente: elaboración propia.

Tabla 5: componentes principales en trabajadores (2).

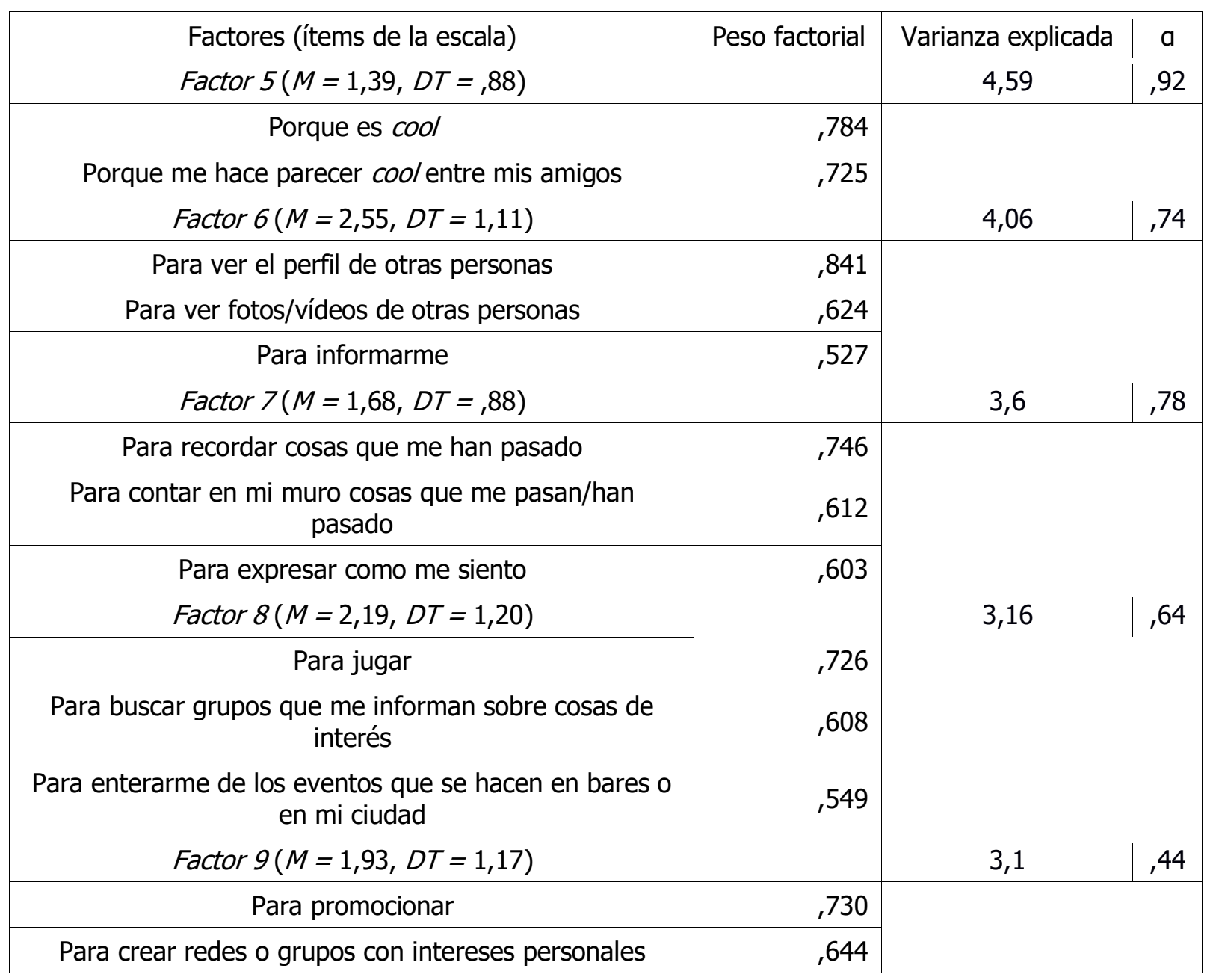

Fuente: elaboración propia.

Como se observa en las Tablas 4 y 5 , se encuentran diferencias estadísticamente significativas respecto al grupo estudiantil. Teniendo en cuenta el resultado que correlacionaba una menor satisfacción a mayor edad, las personas trabajadoras están menos satisfechas con el uso del Facebook $(d=0,53, t=3,33, p .=, 002)$. 
También tienen menor número de contactos $(\mathrm{d}=0,43, \mathrm{t}=2,65, \mathrm{p} .=, 009)$ y disminuyen el tiempo de una conexión puntual $(d=, 67, t=2,25, \mathrm{p} .=, 025)$, pero aumenta en los empleados el hecho de disponer de datos en el celular $(d=, 54, t=-$ $4,2$, p. $<, 000)$.

Respecto a los componentes motivacionales, los empleados puntúan significativamente menos en casi todos los ítems (y factores), excepto en: para ver fotos/vídeos de otras personas; ver el perfil de otras personas; expresar opiniones sobre política o temas sociales; y publicar intereses personales. Estos datos se relacionan con la menor satisfacción comentada anteriormente, pues la media de los trabajadores se sitúa en 6,28 mientras la de los estudiantes era de $7.58(d=0,53)$.

\subsubsection{Comparación entre estudiantes y trabajadores.}

Por último, hay diferencias en el modelo de regresión lineal de cada uno de los grupos, pues aunque los factores más significativos son los mismos, Entretenimiento y Mantener relaciones (cuarto y tercero en estudiantes, y primero y cuarto en empleados), la predicción de la satisfacción es mayor en este último colectivo ( $R^{2}$ ajustado $=, 274$ frente al ,204 en estudiantes), tal y como se puede observar en las Tablas 6 y 7.

Tabla 6: satisfacción. Regresión lineal. Estudiantes.

\begin{tabular}{|r|r|r|r|r|}
\hline Grupo & $\mathrm{R}$ & R cuadrado & R cuadrado ajustado & $\begin{array}{c}\text { Error estándar de la } \\
\text { estimación }\end{array}$ \\
\hline $\begin{array}{r}\text { Estudiantes } \\
\text { a, Predictores: (Constante), Factor 4 (Entretenimiento) y Factor 3 (Mantener relaciones) }\end{array}$ \\
\hline
\end{tabular}

Fuente: elaboración propia.

Tabla 7: satisfacción. Regresión lineal. Trabajadores.

\begin{tabular}{|c|r|r|r|r|}
\hline Grupo & $\mathrm{R}$ & R cuadrado & R cuadrado ajustado & $\begin{array}{c}\text { Error estándar de la } \\
\text { estimación }\end{array}$ \\
\hline $\begin{array}{c}\text { Trabajadore } \\
\text { S }\end{array}$ &, $553^{\mathrm{a}}$ &, 305 &, 274 & 2,217 \\
\hline \multicolumn{2}{|c|}{ a, Predictores: (Constante), Factor 1 (Entretenimiento) y Factor 4 (Mantener relaciones) } \\
\hline
\end{tabular}

Fuente: elaboración propia.

Es decir, aunque los trabajadores tengan una menor satisfacción con Facebook respecto a los estudiantes, esta variable es algo más predecible en ellos a través de los factores relacionados con el entretenimiento y el mantenimiento de las relaciones, algo que puede ser comprendido si se observa la varianza explicada dada por estas dos dimensiones (estudiantes $11 \%$ y trabajadores $43 \%$ ), lo que demuestra que a pesar de que estos factores sean los más relevantes para predecir la satisfacción en ambos grupos, en el caso de los empleados el peso es mucho mayor, pues los 
estudiantes estarían más motivados por usar Facebook en otros aspectos como la Compañía y el Coolness ( $37 \%$ de la varianza explicada), el cual apenas se correlaciona con la satisfacción $(r=, 089 ; \mathrm{p} .=, 226)$.

En definitiva, la similitud entre ambos grupos es que la satisfacción por la plataforma viene determinada mayormente por el entretenimiento y mantenimiento de las relaciones, sin embargo, se diferenciarían en la importancia o peso que estos dos componentes tienen en cada submuestra tal y como se ha observado en los análisis factoriales anteriores.

\subsection{Discusión}

Tras la investigación, queda plantearse si la aplicación estudiada puede concebirse como una moda. Algunos encuestados comentan que ya no utilizan tanto Facebook como al principio en lo que a función social se refiere, y que para este uso, preferían otras plataformas más "íntimas"para relacionarse sentimentalmente y optan por otras aplicaciones como WhatsApp para formar grupos donde intercambiar comentarios y contenidos de diferente índole que los recurridos en Facebook.

Como está ocurriendo en otros países como España, las personas están migrando hacia otras plataformas como Instagram (Marcelino, 2015; PuroMarketing, 29/05/2019), lo que demuestra la posibilidad de que Facebook solo sea una moda. Es decir, que si la red social estudiada se caracterizaba por ser una red de amigos, para algunas personas se había vuelto demasiado social y ahora el uso que le dan es diferente al inicial planteado por Zuckerberg. Pero como demuestra el aumento de usuarios con los años (Statista, 2019), Facebook ha conseguido renovarse gracias a convertirse en una aplicación que va más allá de constituirse principalmente para comunicarse con los amigos. Por ello, es necesario seguir investigando longitudinalmente la evolución de los factores observados con el tiempo, así como estudiar y comparar los resultados con otros países para obtener un mapa más preciso con el que comprender el funcionamiento de los factores analizados.

En lo referente a las diferencias encontradas entre el género, la edad y la profesión u ocupación es interesante tenerlas en cuenta no solo para profundizar en los usos de manera más segmentada, sino que esto también permitiría generar contenidos más específicos tanto publicitarios como propios de la empresa. Así, si por ejemplo se desea enfocar el marketing en las mujeres estudiantes universitarias en una población similar, la publicidad debería direccionarse hacia actividades de entretenimiento y ocio, a la vez que se debería promover la creación de entornos o interfaces más novedosas o cool, pues este tipo de usuarias darían más valor a estos aspectos frente a otros targets. Si bien la gestión de comerciales y de la oferta de actividades tienen muy en cuenta estos factores y otros muchos más gracias al alto y preciso conocimiento de sus usuarios, sí se echaría en falta más personalización con la interface y mayor fomento de actitudes y empleo de las herramientas ofrecidas por Facebook, todo ello de forma más específica según los distintos públicos y conscientes de la evolución de estos. 
También, en los últimos años se ha podido observar que la plataforma también destaca por otros fines como puede ser en el campo de las protestas sociales (Bacallao-Pino, 2016; Chaves-Lopes, 2013; Fernández-Romero y Sánchez-Duarte, 2019; Rodríguez-Polo, 2013; Valenzuela, Arriagada y Sherman, 2012). Los usuarios particulares abarcan funciones diferentes como entretenerse, trabajar, informarse de eventos, del clima, o incluso la compra-venta, donde las redes sociales cada vez influyen más en el comportamiento de compra (Total Retail, 2016). Así, las instituciones y empresas también lo emplean para fines de todo tipo con el consiguiente peligro de que el volumen de negocio dependa de esta red social, "Facebook podrá quitártelo en cualquier momento y de forma totalmente lícita [...] en cuanto esta modifica sus algoritmos (que los modifica) o cambia sus condiciones (que las cambia), tu negocio se puede ir literalmente al garete" (Otto, 01/01/2018).

Por tanto, a la cuestión de si Facebook es una moda, lo único que se puede señalar de momento es que los resultados en cuanto a componentes motivacionales muestran un amplio abanico de usos y que existen diferencias entre colectivos, género o edad. Este panorama permite concebir a la plataforma como una herramienta que se ha sabido adaptar a las necesidades de sus usuarios a medida que estos se han incrementado y evolucionado.

\section{CONCLUSIONES}

Respecto a las dimensiones motivacionales planteadas, los resultados denotan un concepto de Facebook como una herramienta que facilita las actividades cotidianas de unos usuarios enmarcados en un contexto psicológico, evolutivo, social y culturalmente particular. Por ello, se recomienda replicar este tipo de investigaciones comparando diferentes rangos de edad, como pueden ser las personas mayores. Es necesario insertar nuevas respuestas adaptadas al modo de vida de cada generación, tomando en consideración otros indicadores como la personalidad (Ryan y Xenos, 2011), las condiciones familiares, el contexto socio-cultural, político y económico. Por tanto, no es posible hablar de un target único con el que predecir las motivaciones de uso, sino de varios targets con usos y gratificaciones de diversa índole a los que Facebook trata de satisfacer.

Así, se concluye que los usos y gratificaciones (Katz, Blumler y Gurevitz, 1986) observados en esta investigación son predominantemente de carácter social (aceptación) y lúdico, cubriendo parte de las necesidades básicas del ser humano (Maslow, 1970). Además, las múltiples posibilidades que ofrece la plataforma y las diferencias encontradas en los grupos analizados permiten determinar diversas y entremezcladas gratificaciones como, por ejemplo, más enfocadas al entretenimiento y la auto-expresión o reconocimiento en los jóvenes, o bien más direccionadas al mantenimiento de relaciones en el caso de las personas adultas trabajadoras. Es por ello que hay que tener muy presente que los distintos segmentos evolucionan y cambian con el tiempo, y Facebook, consciente de ello, ha sido capaz de ofrecer un amplio abanico de posibilidades capaces de satisfacer las necesidades y adaptarse a las tendencias de cada parte de la población a medida que esta ha ido cambiando. 
Sobre estos datos, se aconseja para futuros estudios que en la medida de lo posible se recurra a las fuentes originales, en este caso, solicitar información al propio Facebook. Esto también solucionaría problemas como establecer una línea base común con la que clasificar los usos de manera concreta y no especulativa. Así, se podrían hacer comparaciones de forma precisa respecto a la cantidad de vídeos que ven en Facebook, el tiempo que pasan chateando o el número exacto de comentarios que cuelgan cada día.

Como se ha deducido, Facebook no es una plataforma más, sino "La plataforma". Se ha convertido en un canal comunicativo de masas, y en algunos casos elitista por excluir de la vida social a aquellos que no quieren formar parte de esta red de amigos.

A su vez, ha creado una serie de hábitos los cuales satisface de manera correcta y bien pensada. Se ha convertido en una agenda diaria virtual, donde uno puede felicitar cumpleaños, mirar las noticias de actualidad, el tiempo en su ciudad o las tareas que el profesor ha enviado para clase; aumentando de esta manera, los efectos típicos de cualquier hábito, especialmente el de la dependencia psicológica (Luengo, 2004; Villafuerte y Mainé, 2005). Los algoritmos utilizados no dejan de sorprender cada año, ya sea para mostrar los posts y comentarios que más pueden interesarle a uno, como aquella publicidad afín a los gustos individuales.

En este campo, Facebook ha ganado adeptos gracias a su componente voyerista, donde muchos usuarios deciden optar por una posición pasiva en sus actividades pero activa a la hora de observar las de los otros. En este sentido, la información proporcionada en muchos casos no es baladí (estado civil, lugar de nacimiento, de residencia, ocupación laboral, estudios realizados, etc.), algo que sobre todo está siendo aprovechado por las empresas de marketing que cada vez más utilizan los datos para proporcionar publicidad más específica y acorde con los heterogéneos clientes, pues no hay que olvidar que Facebook es gratuito, por lo que la empresa debe financiarse de otras maneras como la publicitaria (El Mundo, 01/02/2017).

A esto hay que sumarle la ventaja que tiene Facebook ante la omisión de ciertas responsabilidades sobre comentarios o contenidos ofensivos donde la empresa debería tomar medidas al respecto y "no limpiarse las manos" dejando que sean los usuarios quienes tengan que ser los que denuncien y no la propia plataforma.

En definitiva, se puede concluir que las redes sociales son una herramienta con capacidad para socializar a un grupo de personas de manera paralela a la realidad social física (García-Giménez, 2010), creando a través de ellas sentimientos de pertenencia e identidad que satisfagan las necesidades de expresarse, compartir e interpretar un hecho concreto en relación a un grupo.

Siendo la red social de mayor uso, queda pendiente que la evolución tecnológica y el marketing dispongan de otras herramientas digitales que sustituyan o faciliten cada uno de los servicios que ofrece Facebook, el cual se ha convertido en parte de la vida diaria de millones de personas, grupos, empresas o instituciones a los cuales 
les ha cambiado su manera de comunicarse, de satisfacer sus diferentes necesidades, y especialmente de definirnos de una manera u otra.

\section{REFERENCIAS}

Ahlse, J., Nilsson, F., y Sandström, N. (2020). It's time to TikTok: Exploring Generation $Z$ 's motivations to participate in \#Challenges (Tesis de grado). Jönköping University, Suecia. Recuperado de https://www.divaportal.org/smash/record.jsf ?pid=diva2\%3A1434091\&dswid =5878

Alhabash, S., Park, H., Kononova, A., Chiang, Y., y Wise, K. (2012). Exploring the motivations of Facebook use in Taiwan. Cyberpsychology, Behavior, and Social Networking, 15(6), 210-230. https://doi.org/10.1089/cyber.2011.0611

Bacallao-Pino, L. (2016). Redes sociales, acción colectiva y elecciones: los usos de Facebook por el movimiento estudiantil chileno durante la campaña electoral de 2013. Palabra Clave, 19(3), 810-837. Recuperado de https://palabraclave.unisabana.edu.co/index.php/palabraclave/article/view/5603

Boyd, D. M., y Ellison, N. B. (2007). Social network sites: Definition, history, and scholarship. Journal of Computer-Mediated Communication, 13(1), 210-230. https://doi.org/10.1111/j.1083-6101.2007.00393.x

Callejo-González, J. J. (2019). Percepción y uso de las redes sociales entre adolescentes. Una aproximación a través de Instagram (Tesis de grado). Universidad de Valladolid, España.

Chaves-Lopes, G. (2013). Las redes sociales y los nuevos flujos de programación: un análisis de la cobertura de Al Jazeera sobre la primavera árabe. Palabra Clave, 16(3), 789 - 811. https://doi.org/10.5294/pacla.2013.16.3.5

Cohen, J. (1988). Statistical power analisis for the behavioral sciences (2a ed). New York: Academic Press.

Colás-Bravo, P., González-Ramírez, T., y de Pablos-Pons, J. (2013). Juventud y redes sociales: motivaciones y usos preferentes. Comunicar, 20(40), 15-23. https://doi.org/10.3916/C40-2013-02-01

DataReportal (2020). Digital 2020: Ecuador. Datareportal.com. Recuperado de https://datareportal.com/reports/digital-2020-ecuador?rq=ecuador

Dávalos, N. (14/03/2020). 13 millones de personas tienen redes sociales en Ecuador. Primicias.es. Recuperado de https://www.primicias.ec/noticias/tecnologia/13millones-personas-redes-sociales-ecuador/

El Mundo (01/02/2017). Facebook ganó 9.509 millones en 2016, un $177 \%$ más que en 2015. El Mundo.es. Recuperado de 
Jiménez-Sánchez, A. y Vayas Ruiz, E C. Dimensiones motivacionales en Facebook en estudiantes y trabajadores universitarios del Ecuador

https://www.elmundo.es/economia/2017/02/01/589266c622601d790e8b45b7.htm I

Facebook-Newsroom (2019). Facebook-Newsroom. Facebook.com. Recuperado de https://newsroom.fb.com/company-info/

Facebook para empresas (2019). Marketing en Facebook. Facebook.com. Recuperado de https://www.facebook.com/business/marketing/facebook

Fernández-Romero, D., y Sánchez-Duarte, J. M. (2019) Alianzas y resistencias feministas en Facebook para la convocatoria del 8M en España. Convergencia Revista Ciencias Sociales, (81), 1-21. https://doi.org/10.29101/crcs.v26i81.11943

Flanagin, A. J., y Metzger, M. J. (2001). Internet use in the contemporary media environment. Human Communication Research, 27(1), 153-181. https://doi.org/10.1111/j.1468-2958.2001.tb00779.x

García, A., López de Ayala, M. C., y Gaona, C. (2012). A vision of uses and gratifications applied to the study of Internet use by adolescents. Comunicación $y$ Sociedad, 25(2), 231-254. Recuperado de https://dialnet.unirioja.es/servlet/articulo?codigo $=4462019$

García-Giménez, D. (2010). Redes sociales: posibilidades de Facebook para las bibliotecas públicas. BiD: textos universitaris de biblioteconomia i documentació, 24. Barcelona: Universitat de Barcelona. Facultat de Biblioteconomia i Documentació. https://doi.org/10.1344/105.000001547

García-Guerrero, J. (2015). E-commerce Day, Julio 2015. Ecuador: INEC. Recuperado de $\quad$ http://www.ecuadorencifras.gob.ec/documentos/web-inec/boletin/Ecommerce.pdf

Gutierrez, T. (19/09/2013). Las claves del éxito de Facebook, según Zuckerberg. Alto Nivel. Recuperado de https://www.altonivel.com.mx/38160-las-claves-del-exitode-facebook-segun-zuckerberg/

Hedges, L.V., y Olkin, I. (1984). Nonparametric estimators of effect size in metaanalysis. Psychological Bulletin, 96(3), 573-580. Recuperado de https://psycnet.apa.org/buy/1985-11217-001

Hutchinson, A. (2016). New comScore Traffic Report Underlines the Strength of Facebook, Rise of Snapchat. Social Media Today. Recuperado de https://www.socialmediatoday.com/social-networks/new-comscore-traffic-reportunderlines-strength-facebook-rise-snapchat 
Igartua, J. J., y Rodríguez de Dios, I. (2016). Correlatos motivacionales del uso y la satisfacción con Facebook en jóvenes españoles. Cuadernos.info, (38), 107-119. https://doi.org/10.7764/cdi.38.848

Jiménez-Sánchez, Á., Ortega-Mohedano, F., Vayas-Ruiz, E., Martínez-Bonilla, C., y Lavín, J. M. (2019). Generation $Z$ versus adults in the Ecuadorian digital integration from 2015 to 2019. Proceedings of the Seventh International Conference TEEM'19, October, 885-890. León, España. https://doi.org/10.1145/3362789.3362856

Joinson, A. (2008). 'Looking at', 'looking up' or 'keeping up with' people? Motives and uses of Facebook. Chi 2008, Apri(5-10), 1027-1036. Recuperado de https://digitalwellbeing.org/downloads/Joinson Facebook.pdf

Katz, E., Blumler, J. G., y Gurevitch, M. (1986). Usos y gratificaciones de la comunicación de masas. En M. de Moragas (eds.), Sociología de la comunicación de masas. II. Estructura, funciones y efectos (pp. 127-171). Barcelona: Gustavo Gili.

Kim, B., y Kim, Y. (2019). Facebook versus Instagram: How perceived gratifications and technological attributes are related to the change in social media usage. The Social Science Journal, 56(2), 156-167. https://doi.org/10.1016/j.soscij.2018.10.002

Kim, Y., Sohn, D., y Choi, S. M. (2011). Cultural difference in motivations for using social network sites: A comparative study of American and Korean college students. Computers in Human Behavior, 271), 365-372. https://doi.org/10.1016/j.chb.2010.08.015

Lee, S. J. (2009). Online communication and adolescent social ties: who benefits more from Internet use? Journal of Computer-Mediated Communication, 14(3), 509-531. https://doi.org/10.1111/j.1083-6101.2009.01451.x

Luengo, A. (2004). Adicción a Internet: conceptualización y propuesta de intervención. Revista Profesional Española de Terapia Cognitivo-Conductual, 2, 2252. Recuperado de http://www.jogoremoto.pt/docs/extra/BL5L6u.pdf

Marcelino, G. V. (2015). Migración de los jóvenes españoles en redes sociales, de Tuenti a Facebook y de Facebook a Instagram. La segunda migración. ICONO14, 13(2), 48-72. https://doi.org/10.7195/ri14.v13i2.821

Maslow, A. (1970). Motivation and Personality (2nd ed.). New York: Harper and Row.

Mejía, J. C. (02/05/2017). Estadísticas de redes sociales: Usuarios de Facebook, Instagram, LinkedIn, Twitter, Whatsapp y otros + infografía. Blog Juan Carlos Mejía Llano. Recuperado de https://www.juancmejia.com/marketing- 
Jiménez-Sánchez, A. y Vayas Ruiz, E C. Dimensiones motivacionales en Facebook en estudiantes y trabajadores universitarios del Ecuador

digital/estadisticas-de-redes-sociales-usuarios-de-facebook-instagram-linkedintwitter-whatsapp-y-otros-infografia/

Morais-Martín, D. (2020). TikTok. Análisis de la irrupción de la nueva Red Social en Internet: análisis de las claves de su éxito y sus posibilidades (Tesis de grado). Universidad de Valladolid, España.

Nadkarni, A., y Hofmann, S. G. (2012). Why do people use Facebook? Personality and Individual Differences, 52(3), 243-249. https://doi.org/10.1016/j.paid.2011.11.007

Otto, C. (01/01/2018). Cuando tu negocio depende de Facebook: los sectores que luchan por esquivar la ruina. El Confidencial, Tecnología. Recuperado de https://www.elconfidencial.com/tecnologia/2018-01-01/facebook-fake-newsvirales-videojuegos-ecommerce 1499213/ [13 de mayo de 2020]

Papacharissi, Z., y Mendelson, A. (2011). Toward a new(er) sociability: Uses, gratifications and social capital on Facebook. En S. Papathanassopoulos (eds.), Media Perspectives for the 21st Century (pp. 212- 230). New York, NY: Routledge.

Papacharissi, Z., y Rubin, A. M. (2000). Predictors of Internet use. Journal of Broadcasting \& Electronic Media, 44(2), 175-196. https://doi.org/10.1207/s15506878jobem4402 2

Pardo, A. y San Martín, R. (1998). Análisis de datos en psicología II. Madrid:Pirámide.

Park, N., Kee, K. F., y Valenzuela, S. (2009). Being immersed in social networking environment: Facebook groups, uses and gratifications, and social outcomes. Cyberpsychology \& Behavior: The Impact of the Internet, Multimedia and Virtual Reality on Behavior and Society, 12(6), 729-733. https://doi.org/10.1089/cpb.2009.0003

PuroMarketing (29/05/2019). Facebook es la culpable directa de que sus usuarios pasen menos tiempo en la plataforma. Puromarketing.com. Recuperado de https://www.puromarketing.com/16/32171/facebook-culpable-directa-usuariospasen-menos-tiempo-plataforma.html

Raacke, J., y Bonds-Raacke, J. (2008). MySpace and Facebook: Applying the uses and gratifications theory to exploring friend-networking sites. Cyberpsychology \& Behavior, 11(2), 169-174. https://doi.org/10.1089/cpb.2007.0056

Rodríguez-Polo, X. R. (2013). Bloqueo mediático, redes sociales y malestar ciudadano. Para entender el movimiento español del 15-M. Palabra Clave, 16(1), 45-68. https://doi.org/10.5294/pacla.2013.16.1.2

Ryan, T., y Xenos, S. (2011). Who uses Facebook? An investigation into the relationship between the Big Five, shyness, narcissism, loneliness, and Facebook 
Jiménez-Sánchez, A. y Vayas Ruiz, E C. Dimensiones motivacionales en Facebook en estudiantes y trabajadores universitarios del Ecuador

usage. Computers in Human Behavior, 27(5), 1658- 1664. https://doi.org/10.1016/j.chb.2011.02.004

Salzinger, S., Antrobus, J., y Hammer, M. (2015). The First Compendium of Social Network Research Focusing on Children and Young Adult: Social Networks of Children, Adolescents, and College Students. Hoboken: Taylor and Francis.

Salzman R. (2015). Understanding social media use in Latin America. Palabra Clave, 18(3), 842-858. https://doi.org/10.5294/pacla.2015.18.3.9

Sheldon, P. (2008). Student favorite: Facebook and motives for its use. Southwestern Mass Communication Journal, 23(2), 39-53. Recuperado de https://mafiadoc.com/queue/student-favorite-facebook-and-motives-for-its-useebsco 59bd541c1723ddeeeb1cef91.html

Sheldon, P., y Newman, M. (2019). Instagram and American Teens. Understanding motives for Its use and relationship to excessive reassurance-seeking and interpersonal rejection. The Journal of Social Media in Society, 8(1), 1-16. Recuperado de https://www.thejsms.org/tsmri/index.php/TSMRI/article/view/423

Smock, A. D., Ellison, N. B., Lampe, C., y Wohn, D. Y. (2011). Facebook as a toolkit: a uses and gratification approach to unbundling feature use. Computers in Human Behavior, 276), 2322-2329. https://doi.org/10.1016/j.chb.2011.07.011

Spiliotopoulos, T., y Oakley, I. (2013). Understanding motivations for facebook use: Usage metrics, network structure, and privacy. En Proceedings of the 2013 ACM annual conference on Human Factors in Computing Systems - CHI '13 (pp. 3287 3296). https://doi.org/10.1145/2470654.2466449

Statista (2019). Facebook: number of monthly active users worldwide 2008-2019. Statista.com. Recuperado de https://www.statista.com/statistics/264810/numberof-monthly-active-facebook-users-worldwide/

Tarullo, R. (2020). ¿Por qué los y las jóvenes están en las redes sociales? Un análisis de sus motivaciones a partir de la teoría de usos y gratificaciones. Revista Prisma Social, (29), 222-239. Recuperado de https://revistaprismasocial.es/article/view/3558

Total Retail (2016). They say they want a revolution. Total Retail 2016. Recuperado de https://www.pwc.com/gx/en/retail-consumer/publications/assets/total-retailglobal-report.pdf

Urista, M., Dong, Q., y Day, K. (2009). Explaining why young adults use MySpace and Facebook through uses and gratifications theory. Human Communication, 12(2), 215-229. Recuperado

de http://citeseerx.ist.psu.edu/viewdoc/summary?doi=10.1.1.568.9846 
Valenzuela, S., Arriagada, A., y Scherman, A. (2012). The social media basis of youth protest behavior: the case of Chile. Journal of Communication, 62(2), 299-314. https://doi.org/10.1111/j.1460-2466.2012.01635.X

Villafuerte, A., y Mainé, L. (2005). Personalidad, hábitos de consumo y riesgo de adicción al Internet en estudiantes universitarios. Revista de Psicología, 23(1), 65112. Recuperado de http://www.redalyc.org/pdf/3378/337829529003.pdf

We are social y Hootsuite (30/01/2019). Digital 2019 Global Digital Overview (January 2019). datareportal.com. Recuperado de https://datareportal.com/reports/digital-2019-global-digital-overview

20minutos.es (18/05/2012). Facebook hace historia con su estreno en Bolsa pese a arrancar con una leve subida del 0,6\%. 20minutos.es, Tecnología. Recuperado de http://www.20minutos.es/noticia/1457200/0/facebook/bolsa/zuckerberg/

\section{AUTORES:}

\section{Álvaro Jiménez Sánchez}

Doctor en Comunicación por la Universidad de Salamanca. Actualmente profesorinvestigador de la carrera de la Facultad de Jurisprudencia y Ciencias Sociales perteneciente a la Universidad Técnica de Ambato (Ecuador). Dirige varios proyectos en edu-entretenimiento. Ha publicado múltiples investigaciones en el campo de la comunicación y las nuevas tecnologías. Director del grupo de investigación "Comunicación, Sociedad, Cultura y Tecnología".

al.jimenez@uta.edu.ec

ORCID ID: https://orcid.org/0000-0002-4249-8949

\section{Eliza Carolina Vayas Ruiz}

Doctora en Ciencias de la Comunicación. Actualmente profesora-investigadora de la Carrera de Comunicación perteneciente a la Universidad Técnica de Ambato (Ecuador). Coordinadora de investigaciones financiadas en el área de comunicación para el Desarrollo, violencia de género y salud. Ha publicado diversas investigaciones en revistas y editoriales de prestigio. Coordinadora del Programa de difusión "Divulga Ciencia-UTA".

elizacvayasr@uta.edu.ec

ORCID ID: https://orcid.org/0000-0002-3012-144X 